\title{
CDISC Protocol Study Purpose Response Terminology
}

National Cancer Institute

\section{Source}

National Cancer Institute. CDISC Protocol Study Purpose Response Terminology. NCI

Thesaurus. Code C147067.

Terminology associated with the study purpose response codelist of the Clinical Data Interchange Standards Consortium (CDISC) protocol entities. 